In using the canula, however, there is considerable danger of displacing the ligature ; and, in tightening the ligature, it often happens that it is cut by the edge of the canula. To obviate these and other objections, as well as to facilitate the tying of the ligature, $I$ have contrived an instrument which, although very simple in its construction, will, I trust, be found very effectual. This instrument is represented below. The two rods, armed with a ligature, are to be held together and passed under the arch of the pubis, upon the superior surface of the tumour, to its neck; one rod is then to be carried to the right hand and the other to the left, until they meet at the inferior part of the vagina; the ring is next to be passed over the ends of the rods, and pushed along, till it arrives nearly at the upper end of the instrument; the screw is then to be inserted, the ligature drawn tight, and the operation is completed.

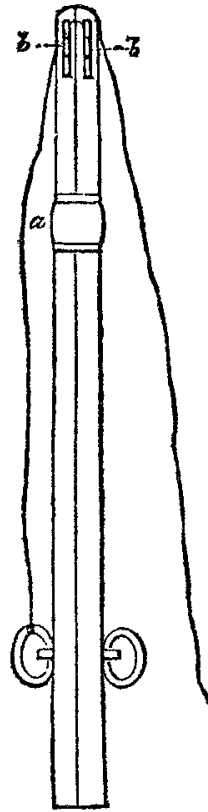

Explanation.-Polypus rods (ten inches in length) united. The ring, $a$, is introduced in order to prevent the possibility of the rods being separated while the ligature is tightened. The ligature works upon two small pulleys which are contained in the eyes of the instrument at $b, b$. By this contrivance the ligature moves with the greatest freedom, and all danger of cutting or chafing it is completely obviated.

A similarly constructed instrument, but of smaller dimensions, could often be successfully employed in the extirpation of polypi from the nares.

With this instrument Mr. Lloyd Peck, of Newmarket, last year removed a polypus from the cervix uteri weighing more than a quarter of a pound, with the greatest facility, after he had been foiled in an attempt to extirpate it with a threerodded instrument, precisely similar in its construction to that described by $\mathrm{Dr}$. Quackenbush.

\section{CASE OF PARAPLEGIA.}

By R. B. Gonham, Esq., of Alderston, near Woodbridge.

The patient, whose name is $\mathbf{J}$. Thompson, thirty-one years of age, having previously enjoyed excelle..t health, was attacked on Thursday, October the 5th, whilst at his work, namely, collar-making, with a feeling of fulness about the lower end of sacrum, and my partner, Mr. Kett, of Hollesley, supposing it arose from a constipated state of his bowels, gave him three strong cathartic pills, which had a very slight effect. He felt relieved, and continued his work for the two following days, but on Saturday, October 7 th, he lifted a sack of potatoes into a cart without any help, which effort was followed by a momentary and slight pain in the lumbar region, and a feeling, on walking home afterwards, a distance of two miles, as if he had not the power of moving one leg before the other. On Sunday, the 8th, he visited me at my surgery, in the village where he and $I$ reside, and the symptoms were, pulse slightly full and regular; tongue moist; no pain in any part of the body; abdomen distended; urine passed naturally, and in the usual quantity; no cephalalgia; no fever, but the loss of power of motion increasing, the bowels having been but slightly relieved, $I$ ordered him a strong purging mixture, and desired him to go home and keep quiet for that day, resting himself in the horizontal position.

9. The patient sent for me with the symptoms very much aggravated as regarded the loss of sensation and power of motion; no action on the bowels. Croton oil pills, one minim in each, one directly and the other in two hours. No effect. Calomel, six grains and jalap twenty grains, immediately. Continue the mixture. Ordered an enema, which the patient would not allow to be administered. Still no effect.

10. Powder, pill, and mixture, as yesterday, together with an injection, which I insisted upon. These measures had the desired effect, and a copious discharge of feculent and scybalous matter followed. Now commenced the incontinence of fæces.

11. Finding the patient's power of motion and sensation quite gone in the lower half of the body, I employed venesection to twenty ounces, a strong rubefacient embrocation to the back and both thighs; repeated the cathartic mixture at intervals of six hours during the day, and in the evening applied a large blister, about two inches wide, from the middle of the back to the end of the sacrum, and kept it open with savine cerate for six weeks, administering the iodide of potassium in five-grain doses every four hours, with the idea of producing absorption of any effused fluid.

12. No pain in the head or dimness of sight; the bowels slightly relieved, but involuntarily, and the urine passes away withont any assistance from art; neither the abdomen nor the bladder are distended. Continue the iodide of potass.

13. Remedies continued, with the addition of calomel and opium, a grain and quarter, every six hours.

14. The same medicines, and the patient's symptoms the same.

15 and 16 . The same plan as heretofore, and no improvement in the symptoms.

17. On this day, finding the patient complaining of slight pain in the lumbar region, I cupped him, and continued the medicines.

18. I found him still suffering from slight pain, and would have repeated the cupping but he would not allow it to be done, as he said he felt more loss of power from it. I continued the iodide and calomel.

19. I gave him two more croton oil-pills, which had the desired effect.

20 and 21 . The same remedies were employed, and some senna and salts given him regularly every morning.

22. My patient complained of a great deal of pain in the blaclder, which, on examination, I found distended, he not having passed any water during the night. I gave him an opiate, and introduced the catheter, and drew away about a pint and a half of urine, presenting the peculiar scent of paraplegic cases. This afforded relief. The iodide and calomel were proceeded with.

23. I again introduced the catheter, previously giving an opiate, as the pain and distention had returned, although a great quantity of urine had dribbled away, and requested him to keep the instrument in the bladder, a gum-elastic one, but after allowing it to remain in two hours he withdrew it, as he said it caused him pain, and he would much rather have it introduced as occasion might require. From this day until the 2 sth of October I introduced it every day, drawing away sometimes two pints, at others only a pint, highly offensive. I this day began the strychnine, one-twelfth of a grain, according to the formula of Magendie, three times a day, from this time to the 24th day of January, 1844, gradually increasing to every four hours, and when its effects became severe intermitting its use for a day or two.

25. I applied the tartarised antimony all the way down the spine, repeating it each time the crop of pustules healed, for two months. From the commencement of the strychnine to the 2 th of January there was a gradual improvement, the bladder recovered its power of retaining and expelling its urine, and by degrees the sphincter ani regained its office, and now $I$ am happy to say the man is at work, which he commenced about a fortnight since, and appears to be improving in every respect day by day. Electricity was also employed in this case in the month of December, by isolating the patient, and taking sparks from him, but he begged to have its use discontinued as it began, afier a fortnight's employment, to produce violent spasmodic contractions; but I persevered for two weeks longer, but only twice during the seven days instead of every day as I first began with.

I imagine that there was either an extravasation of blood or an effusion of serum into the vertebral canal, pressing upon the spinal marrow, produced by the great exertion of lifting the sack of potatoes into the cart, and the want of any symptoms of inflammation or even pain upon pressure along the whole course of the spine, warrants 
the conclusion that the opinion was correct, and the success attending the treatment has the same tendency. Below the umbilicus there was not the slightest feeling, whilst immediately above there was perfect sensation. The patient's expression was, "he felt whole above the navel." I considered the pain in the bladder rather a good symptom, supposing that from the effect of the calomel and opium with the iodide of potassium, that absorption had begun, and although $I$ continued the last named medicine for a few days after the beginning of the strychnine, still I fancy I am justified in thinking that the drug would not have had so excellent an effect if it had been prescribed before.

\section{A CASE OF PREGNANCY MISTAKEN AND TREATED FOR OVARIAN DROPSY.}

By P. Mageniss, Esq., M.R.C.S.

ON the 9th of August, 1843, I was requested to see Mrs. S., thirty-three years of age; married twelve years ; states that she has menstruated regularly both before and since her marriage, until last April, soon after which period she became alarmed at the unusual bulk of her abdomen and other slight constitutional disturbances,having at first attributed her suffering to suppressed catamenia from cold, and having tried some domestic remedies without any relief, she now became more alarmed from the observations of her friends, who induced her to consult a physician in the neighbourhood about three months since, and has repeated her visits twice a week until within the last eight days. She is now unable to move from her couch. She states that she has been bled both locally and generally, freely purged, diuretics administered, mercury given to salivation, and blisters, rendered perpetual by stimnlating dressings. During all this proceeding the only investigation instituted by this physician was, she stated, the examination of the tongue and pulse. The enlargement of the abdomen still increasing, and the patient's strength becoming much weaker, something having been said about the operation, tapping (not percussion) likely to become necessary, the patient did not repeat her visit to the doctor. As I had attended her in the early part of the same year for abscess of the left labia pudendum, which was freely opened and soon got well, she became anxious that I would see her again, and, on my arrival, finding that she was much depressed in health and strength from irritative fever, I prescribed some sedative medicines with nutritive diet. In a few days she was sufficiently recovered, and the blistered surface healed so far, as to enable me to make an examination, and I found that pregnancy had arrived at about the seventh month; the motion of the child in utero was sufficiently strong after the investiration to be felt through the abdominal parietes. Independent of the aid of the stethoscope, which gave distinctly the placental murmur as well as the fotal heart-sound, having satisfied myself by the collective evidence that pregnancy was present, I endeavoured to persuade her that her illness arose from that cause, and not from ovarian dropsy, as she had been before told by the physician, and I had some difficulty in inducing her to think with me; and, to aford her further satisfaction, I requested that $\mathrm{Dr}$. Heming should see her, and he, with his usual care in investigating disease, assured our patient that she was then pregnant. Her mind being: now at ease that no operation would be necessary for her cure, her health became much improved, and on the morning of October 25, 1813 , after a painful labour of fourteen hours' duration, the diagnosis was confirmed by our patient giving birth to a well-developed male child, which died a few days since of hydrocephalus. No bad symptoms supervened upon parturition, nor were any symptoms of ovarian disease present. The patient continues in good health up to this time.

Remarks. - The above case fully proves the importance of making careful examinations to ascertain the nature and seat of disense, when it does exist, before we adopt any serious proceeding for its cure, and when we consider the amount of obloquy such mistakes is calculated to bring upon the profession, it will well illustrate the necessity of making careful examinations, even at the risk of censure from a fastidious patient.
NECROSIS OF THE LOWER MAXILLA AFTER SCARLATINA.

By Rrchard Brown, Esq.

A chrLd, four years old, was brought to me last week suffering from necrosis of the inferior maxilla. The father told me she had a severe attack of scarlatina some time since, and that the lower jaw shortly after became diseased; he had taken her to several medical men, who advised him to wait until the diseased mass became loosened; this was the case when I saw the child, but no attempt had been made to remove it. The sufferings of the little patient urged me at once to do something to alleviate the distressing symptoms, and I forthwith proceeded to remove the necrosed portion.

The alveolar process in the centre was movable but fixed at either extremity by the anterior molar teeth, which I was obliged to remove with one of the bicuspids before I could proceed further. Having done this, I endeavoured to elevate all that was dead, but $I$ experienced some difficulty in separating it; this, however, I effected after a few attempts, without using much force. Some hæmorrhage took place, but not to an alarming amount, and was controlled by pressure with dossils of lint. The child remained with me some time after the operation, and, the bleeding having quite ceased, left, and in $\mathbf{a}$ much more comfortable condition, for it was then enabled to close its mouth, which it could not do before. The father had been led to expect the child would get a new supply of teeth, but I made bold to tell him if it did they would probably be artificial. I have not seen the little patient since, and as it was travelling the country no doubt it has fallen into other hands.

The portion removed was a mass of alveolar proeess which had occupied the space from one anterior molar tooth to the other, and consequently contained the bicuspids and incisors as well as the two anterior molars. The two molar and one bicuspid I removed during the operation; the other bicuspid remained in its socket, but quite loose. The incisors had from time to time been extracted by the child.

\section{By H. Haynes Walton, Esq., Surgeon to the Central} London Ophthalmic Institution, \&c.

IN consequence of having seen in THE LANCET, of March the $23 \mathrm{~d}$, the narrative of a case of hydrophobia, by Mr. Bateman, I have been induced to copy from my note-book, for publication, the particulars of a like case, which came under my notice when house-surgeon to St. Bartholomew's Hospital. Although I am unable to furnish such an extensive post-mortem detail as $\mathbf{M r}$. Bateman has given of his patient, I think, however, the case to be worthy of record, inasmuch as we are totally ignorant of the nature and treatment of that formidable malady; and that, from a collection of well-marked cases, something of practical importance may be discovered or confirmed.

David Read, aged eight, an intelligent and healthy lad, was brought to the surgery at St. Bartholomew's Hospital, on the lith of October, 1811 , by his sister, who was but a few years older, in consequence of injuries received on the eyelid and knee, from having been knocked down by a strange dog, whilst at play with his school-fellows.

Upon examination, I found a superficial wound, or abrasion, on the integuments of the upper eyelid of the right eye, parallel with the cilia, and about three-quarters of an inch in length, and a quarter of an inch in breadth; there was scarcely any bleeding from it, not the slightest swelling, nor did it appear to cause the least inconvenience This was just half an hour after the accident. On the integuments over the patella of the left knee, there was an ecchymosis, about the size of a shilling, with a slight swelling. The little patient said that whilst playing with his school-fellows, he was suddenly knocked down by a large dog that jumped on his side, but that where the animal came from, or what became of him, he could not tell, neither could he give any information of the probable condition of the animal's health. It was impossible to elicit any satisfactory evi- 